\title{
On mindset, mental preparation and chasing breakthroughs: A physician-scientist trainee's perspective
}

\author{
Karan Joshua Abraham
}

Faculty of Medicine and University of Toronto, Toronto, ON

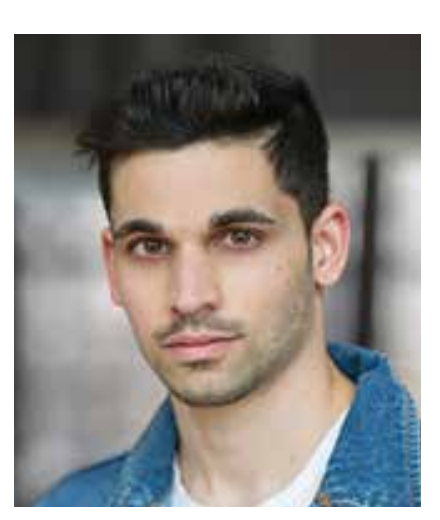

\begin{abstract}
BIO
Karan ("Josh") Abraham is a physician-scientist trainee in the University of Toronto MD-PhD program. He is a Vanier Scholar, Ruggle's Innovation Award winner, Adel S. Sedra Distinguished Graduate Award recipient, and is currently president of the Clinician-Investigator Trainee Association of Canada (CITAC). In his PhD work, he uses molecular, genetic and cell biological approaches to uncover mechanisms that preserve the integrity of the genetic code and sustain the protein synthesis capacity of cells. He hopes to lead a basic research program that will advance scientific knowledge to better understand and treat human disease, and to one day become a leading ambassador for Canadian biomedical research.
\end{abstract}

\begin{abstract}
1 s dual practitioners of science and medicine, we have a moral obligation to better prepare ourselves and our trainees to deliver and translate important discoveries. Here, I reflect on specific mindsets that add fuel to a lifelong quest for elite performance, ultimately preparing us to make scientific breakthroughs in our fields.
\end{abstract}

What do physician-scientists share with leading athletes and business moguls? Elite athletes are remembered by their physical feats (e.g., Bannister's first sub-4minute mile); leading entrepreneurs by their commercial victories (e.g., Gate's Microsoft). As investigators, we are measured by our discoveries, and their impact on the lives of our patients. Notwithstanding the difference in end-product, I believe the path of every elite performer is built on the same foundations - sacrifice, preparation and a commitment to continually advance our craft; a path that demands more than most people are able - or willing - to commit.

I find it surprising, and somewhat ironic, that the "science" of raising performance is underdeveloped and underutilized in the practice of medicine and science. In the typical institutional and educational setting, discourse on performance is limited to measures (productivity, awards, etc.) or surface-level enablers (time management skills, knowledge acquisition, etc.), without an adequate exploration of the deeper values that drive preparation and progress. Take Fleming's groundbreaking discovery of penicillin; a historical breakthrough borne from the instinct and conviction to pursue an observation most would surely have ignored. I am referring to deeper drivers of performance- imagination, courage and resilience; qualities that truly underlie a breakthrough result. Yet, how much attention do we devote to cultivating and coaching these qualities?

In the quest to develop a more holistic blueprint for performance, there is much we can learn from business and sport. In these result-oriented domains, every detail of performance is accounted for; no stone is left unturned. Elite athletes now incorporate detailed prehabilitation, nutrition and recovery programs, into more conventional routines of athletic preparation. On a deeper level, sport psychology and mindset preparation aims to raise performance by fostering empowering beliefs and attitudes that complement physical prowess 
and skill. This "unseen work" provides a less tangible but no less valuable foundation upon which outstanding performance is built. Can we adopt a similar blueprint? For inspiration, I look to my mentors; whose approaches have helped me add a new dimension to not just my level of application - but also my enjoyment - in the pursuit of discovery. Below, I share my interpretation of the four most valuable tenets of their collective philosophies: understanding commitment, balancing pragmatism with imagination and courage, prioritizing growth over comfort and performance built on a foundation of wellness. I can say with conviction that these lessons that have contributed more to my individual development than any workshop, textbook or skill ever have.

\section{Understanding commitment}

There is a key distinction between interest and commitment that every aspiring clinician-scientist must learn. Interest implies making the effort only when it is convenient, and, therefore, interest can be lost as quickly as it is gained. Commitment, in contrast, demands that every reasonable measure is taken to master one's craft, even when challenging circumstances may justify otherwise. My PhD supervisor once proclaimed that the success or failure of our PhDs lay entirely in our hands; not as a way of absolving himself of his own obligations, but rather as a reminder that commitment begins by assuming full ownership of the task at hand. Practically, the internalization of this philosophy promotes self-reliance; self-reliance drives initiative; and initiative begets results. A leader who can coach a group of individuals to buy into this ethos is poised to advance the team's individual and collective prospects for a breakthrough discovery. Thus, commitment, and not interest, leads to breakthroughs.

\section{Balancing pragmatism with imagination and courage}

In clinical practice, pragmatism and risk reduction are critical imperatives that maintain patient safety. Yet we tend to overuse these mental muscles at the expense of creativity and imagination, particularly as we mature in our professions. In the quest for discovery and innovation, creativity remains one of our most potent tools; I have learned to view it as a skill that can be coached rather than a quality that is innate. This I credit to the former director of our MD-PhD program, who was a strong advocate of dedicating time to conscious, unfettered creative practice. In a typical session, the brain is unleashed against a clinical or scientific problem, and imagination given full license to roam. This form of mental preparation enhances problem-solving and breeds enthusiasm for discovery. What about courage? It takes courage to tackle a difficult problem, it takes bravery to examine current dogma with a critical eye, though arguably not as much as it takes to refute your supervisor's hypothesis! Like imagination, courage needs to be rehearsed, and rewarded, in the day-to-day practice of our profession. Thus, through the conscious practice of creativity and courage, performance can be elevated, and the pursuit of discovery emboldened.

\section{Prioritizing growth over comfort}

No circumstance provides a greater opportunity to craft resilience and maximize progress than adversity. Every challenge I faced in my PhD, though exasperating in the moment, also precipitated rapid personal and professional growth. It can be instinctual to perceive adversity as a personal indictment of a body of work or one's career potential, but it is also completely counterproductive. My PhD taught me to reexamine these moments with equipoise. A rejection becomes a helpful report card; a failed experiment becomes a stepping stone to the truth. Performed often enough, the brain learns to surrender its self-limiting associations to challenging situations and exchange them for beneficial ones. Adversity is no longer feared as a demoralizing indictment; instead it is welcomed as a precursor for growth. This mental reframing not only accelerates learning, it provides equanimity, strengthening the belief that future challenges can also be overcome. It may be easier to evade challenge rather than to invite it, and simpler to avoid adversity rather than to confront it; but ultimately, a breakthrough cannot be engineered on weak foundations.

\section{Performance built on a foundation of wellness}

It is essential that aspiring physician-scientists recognize that a holistic and long-term approach to performance is 
built on a foundation of wellness. If our professional obligations are a large and rewarding part of our lives, then our lives outside work provide the license to enjoy and thrive inside it. Performance in a professional setting therefore begins at home; it is sustained through positive relationships, and it can only be enhanced through a more informed and systematic approach to nutrition, recovery, exercise and other forms of wellness promotion. Thus, carefully mapping out and optimizing the individual variables that comprise our collective wellness equations is a prudent investment towards enhanced professional performance and improved prospects for a breakthrough discovery.

Physician-scientist or trainee, I encourage each of you to consider how a deeper examination of beliefs, coupled with a more holistic approach to preparation as outlined by the aforementioned principles, may improve the current paradigm for individual and team performance in the setting of medicine and research.

\section{CORRESPONDENCE TO:}

E-mail karan.abraham@mail.utoronto.ca 